\title{
La naissance de la problématique histologique et l'Ecole Clinique de Paris *
}

\section{Par Othmar Keel}

Dans des travaux antérieurs, nous avons analysé les conditions de la constitution d'une problématique histologique dans les sciences bio-médicales. Nous avons montré que cette problématique s'était formée, pour une part importante, indépendamment des travaux de l'Ecole de Paris (Pinel, Bichat etc...). ${ }^{1}$

Dans la mesure où l'histoire traditionnelle de la médecine et de la biologie a propagé la thèse que le concept de tissu n'avait pu se former qu'avec la leçon de Pinel et de Bichat, on pourrait croire que les travaux antérieurs entrepris à partir d'une problématique tissulaire sont demeurés inconnus ou méconnus en France comme ailleurs. Qu'en est-il réellement?

Nous avons déjà démontré qu'un champ de recherche dans le domaine de l'analyse des tissus s'était constitué à partir d'enseignements comme ceux de Haller $^{2}$, de A. Bonn ${ }^{3}$, des Hunters ${ }^{4}$, de Smyth, etc... et que ces recherches sur les tissus avaient été reconnues comme telles par certains auteurs du XIX ${ }^{\mathrm{e}}$ siècle.

Une analyse plus systématique des textes de l'époque permet de voir d'autres auteurs encore avaient identifié adéquatement les conditions de la formation de la problématique tissulaire, ce qui permet de mieux cerner encore quelles ont été, avant l'Ecole de Paris, les contributions à la constitution de la problématique histologique. Il est d'autant plus nécessaire de rappeler ce point que des ouvrages publiés récemment ${ }^{6}$ perpétuent encore la légende selon laquelle ce serait uniquement avec l'Ecole de Paris, et grâce aux nouvelles idées de Pinel et de Bichat, que serait apparue une approche tissulaire en médecine et en biologie ${ }^{6}$.

Selon plusieurs commentateurs de la fin du XVIII ${ }^{\mathrm{e}}$ et du début du XIX ${ }^{\mathrm{e}}$ siècle, une place très importante dans la constitution de l'anatomie générale revient à J. Hunter. C'est ainsi que, en 1822, Monfalcon écrit: «L'analyse des organes, cette grande pensée, voilà Bichat tout entier.» Monfalcon entend,

\footnotetext{
* Le présent travail est extrait d'un ouvrage devant être publié prochainement. Ce travail a été rendu possible par des subventions du Conseil de recherches en sciences humaines du Canada ainsi que du Fonds National Suisse de la recherche scientifique. Dans le texte qui suit, toutes les traductions en français des citations tirées d'ouvrages en d'autres langues sont de nous.
} 
bien sûr, par l'analyse des organes, leur décomposition en leurs constituants élémentaires ou tissus. Monfalcon ajoute cependant que:

\begin{abstract}
Hunter, avant Bichat, avait observé que les phlegmasies internes affectaient des tissus d'espèce différente, qu'il en existait de particulières aux canaux excréteurs (inflammations des membranes muqueuses), et d'autres qui ont leur siège dans les cavités circonscrites (phlegmasies séreuses); il savait que lorsqu'il existe une violente inflammation abdominale, la maladie est bornée, dans certains cas, à la membrane qui enveloppe et les parois et les viscères de l'abdomen, et, que, dans d'autres, étrangères à ce tissu, elle affecte exclusivement la membrane muqueuse gastro-intestinale. Quelques monographies, celles de Bordeu sur les glandes et le tissu muqueux, ont précédé le travail de Bichat; M.Pinel enfin avait, avant sa publication, classé une grande partie des phlegmasies d'après les tissus qu'elles affectent, et enseigné que l'étude des organes, d'abord sous le rapport de leur structure, puis sous celui de leurs fonctions, est le moyen le plus certain d'acquérir des lumières sur leurs maladies ${ }^{7}$.
\end{abstract}

Selon Monfalcon donc, les travaux de ces auteurs (Hunter, Bordeu, Pinel) ont précédé et rendu possibles ceux de Bichat. Si l'on ajoute à cela - et Monfalcon semble l'ignorer -, que la problématique de Pinel est une retraduction des travaux des médecins et chirurgiens des écoles écossaises et anglaises comme Smyth et les Hunters, on voit déjà que ce n'est pas dans l'Ecole de Paris que s'est formée la problématique histologique. D'autres auteurs font la même analyse que Monfalcon, et notre argument prend d'autant plus de poids que la plupart d'entre eux mentionnent justement l'apport essentiel de Pinel, en ignorant que cette contribution n'est pas la sienne, mais celle de l'école d'Edimbourg et de l'école des Hunters à Londres ${ }^{8}$.

Parmi les auteurs qui ont donné une idée juste des apports à la problématique tissulaire qui sont antérieurs aux travaux de Pinel et de Bichat, on peut mentionner C. Saucerotte, qui a écrit en 1837:

La péritonite elle-même était ignorée, méconnue des praticiens, bien que les recueils de Bonet, de Schenk, de Lieutaud, de Morgagni, renferment plusieurs observations de cette phlegmasie à l'état aigu ou chronique; dans aucun on ne trouve cette lésion décrite sous son véritable nom. Quelques médecins, cependant, éclairés par leurs recherches nécroscopiques, soutinrent que cette fièvre était occasionnée par l'inflammation de l'épiploon et des intestins; ne voyant que le phénomène saillant de la phlegmasie, sans reconnaître l'indépendance pathologique des tissus, vérité dont la découverte était réservée à Hunter, à Pinel, à Bichat. Hunter annonça que la fièvre puerpérale reconnaît pour cause la phlegmasie du péritoine, sans que les intestins y participent. Cette importante assertion n'eut toutefois cours dans les sciences qu'au bout de quelques années, lorsque Walter à Berlin, Pinel et Bichat à Paris, lui eurent donné la sanction de leurs travaux ${ }^{9}$. 
Nous avons avec ce texte un exemple typique d'une demi-reconnaissance du fait qu'il a existé une approche et une problématique tissulaires avant Pinel et Bichat. Premièrement, Saucerotte souligne le fait que déjà Bonet, Schenk, Morgagni, Lieutaud, avaient fait plusieurs observations d'une maladie localisée au niveau d'un tissu comme la péritonite. En second lieu, il affirme que le principe de «l'indépendance pathologique des tissus» a été découvert par Hunter d'abord, mais il semble croire que ce principe a été découvert aussi par Pinel et Bichat indépendamment de l'auteur écossais. Or, comme nous l'avons démontré ailleurs, ce n'est pas indépendamment de Hunter (et de certains autres auteurs comme Smyth, Monro, Baillie, etc...) que Pinel et Bichat ont produit une conception tissulaire de la maladie ${ }^{10}$. En deuxième lieu, Saucerotte semble croire que le principe de l'indépendance pathologique des tissus découvert par Hunter n'a eu cours dans la science que par la sanction des travaux de Walter à Berlin et de Pinel et Bichat à Paris, ce qui est également faux. En effet, cette conception tissulaire de la maladie, et de la fièvre puerpérale en particulier, s'était déjà répandue en Grande-Bretagne même (chez des élèves et des confrères de Hunter comme Smyth, Baillie etc...), ainsi qu'ailleurs en Europe ${ }^{11}$.

Si les travaux d'un Pinel ne sont pas indépendants de ceux de Smyth, on peut dire aussi que plusieurs auteurs avaient indiqué que ceux de Bichat ne sont pas indépendants de ceux de Hunter. Et il ne s'agissait pas d'inconnus, mais d'auteurs aussi importants que Broussais dont les travaux ont connu à l'époque une diffusion considérable. En effet, dans l'Examen des doctrines médicales, et des systèmes de nosologie, Broussais a écrit:

\footnotetext{
Ainsi c'est à cet auteur (Hunter) que nous sommes redevables des premières bonnes distinctions sur le siège des phlegmasies, distinctions dont on a profité pour l'avantage de la science, et qui plus tard ont conduit notre Bichat aux considérations de son Anatomie générale $^{12}$.
}

Par ailleurs, il est intéressant de noter que si certains auteurs ont reconnu qu'il y avait des analogies frappantes entre la problématique de Hunter et celle de Bichat, d'autres ont vu de telles analogies entre les idées de ce dernier et celles de Smyth.

Par exemple, en 1833, le clinicien W. Alison, dans l'article "History of Medicine" de la Cyclopaedia of Practical Medicine considère que la portée scientifique de l'œuvre de Smyth est équivalente pour la médecine à celle des travaux de Bichat: 
Beaucoup a été fait pour ce qui concerne le cours et les effets différents de l'inflammation en tant qu'ils affectent des tissus différents du corps, et il a été établi que même dans sa forme aiguë, l'inflammation s'étend fréquemment, dure longtemps, et produit des lésions décisives dans un tissu sans affecter au moindre degré les autres tissus qui sont dans son voisinage immédiat. Des observations répétées sur les corps de ceux qui sont morts de pleurésie, de bronchite, de péritonite et de dysenterie, et aussi d'inflammations plus externes, ne laissent pas de possibilité de doute sur ce point. Auparavant, le diagnostic des différentes maladies inflammatoires allait rarement plus loin que les organes principalement affectés; maintenant nous considérons la texture affectée en premier comme un des principaux objets de recherche. La variété dans le cours et les effets de l'inflammation que l'on peut observer dans les différents tissus: dans les membranes cellulaires, séreuses, fibreuses et muqueuses, dans les différents viscères parenchymateux, les muscles, les os, etc. fut pour la première fois désigné comme objet particulier d'attention par Bichat en France et par le Dr. Carmichael Smyth en Angleterre ${ }^{13}$.

Paradoxalement, Alison considère donc Smyth comme le Bichat anglais. Il se trompe en ce qu'il croit que les travaux de Bichat ne doivent rien à ceux de Smyth. Ceci vient du fait qu'il ignore que la problématique tissulaire de Smyth a été transmise à Bichat par l'intermédiaire de Pinel. Par ailleurs, Alison semble ignorer que la problématique de Bichat doit beaucoup à Hunter.

Dès 1813, dans son livre Outlines of the Anatomy of the Human body in its sound and diseased state (Edimbourg, 1813), Alexander Monro (tertius) avait déjà clairement laissé entendre que la problématique de Bichat ne pouvait pas être à ce point isomorphe à celle de Smyth, sans qu'il y ait une filiation entre les deux auteurs:

En raison de la ressemblance remarquable que l'on trouve entre les manières de penser, et de nombreux passages de l'essai du Dr. Carmichael Smyth sur l'inflammation publié dans le $2^{\mathrm{e}}$ volume des London Medical Communications, et le Traité des membranes ainsi que l'Anatomie générale de Bichat, je ne peux pas ne pas penser que que le Dr. Smyth était l'auteur du nouveau système qui a ouvert la voie à un examen, d'un niveau plus élémentaire, plus exact et plus philosophique, des structures et des propriétés de nos différents organes, système qui a donné une impulsion si remarquable à l'avancement de la science physiologique et pathologique, que celle-ci a pris effectivement maintenant une nouvelle configuration ${ }^{14}$.

Il vaut la peine, croyons-nous, de signaler que ce texte de Monro avait été remarqué par C. F. Heusinger dans son System der Histologie en 1822:

D'après Al. Monro (Outl. of the Anat. of the hum. body, vol I) C. Smyth aurait exprimé dans les London med. Communications Vol II, dans un traité sur l'inflammation, des vues qui seraient très analogues à celles de Bichat. Je n'ai pas cet écrit sous la main et ne suis donc pas en mesure de juger du bien-fondé ou non de cette assertion ${ }^{15}$. 
Un autre auteur qui a donné une idée juste des conditions de l'élaboration d'une problématique histologique est R.D.Grainger dans ses Elements of General Anatomy:

Une modification des plus importantes dans le mode d'étude de la composition du corps animal, commença à s'imposer rapidement à partir de la première moitié du siècle dernier. A cette période, plusieurs auteurs mirent en évidence l'analogie et les connexions qui existent entre certaines membranes et d'autres parties, que l'on avait considérées antérieurement exclusivement comme autant de structures isolées et indépendantes. Un de ces premiers auteurs est Andreas Bonn, lequel, dans une thèse publiée en 1763 et intitulée De continuationibus membranarum fit plusieurs observations importantes, dont le crédit a généralement été attribué à ses successeurs et, notamment, à Bichat. Quelques années plus tard, la nécessité de prendre en considération les tissus des différents organes fut illustrée dans un excellent travail sur l'Inflammation, qui fut lu par le Dr. Carmichael Smyth, au cours de l'année 1788. Il affirme, que, après avoir observé avec soin les différentes formes d'inflammation, il a été amené à considérer que les principales causes des distinctions spécifiques qu'elles présentent, dépendent de la texture naturelle et de la fonction de la partie enflammée... Ceci semble avoir été le premier essai pour ordonner les symptômes de l'inflammation selon le tissu de l'organe dans lequel la maladie se produit. Vers la même époque, Pinel adopta une méthode à peu près semblable de distinction des maladies selon la structure des solides organiques. Il n'est peut-être aucun événement à l'époque moderne qui ait eu une si grande et si bénéfique influence sur la pratique de la médecine que ce progrès dans la manière de cultiver l'anatomie humaine. Cela a conduit les praticiens de la médecine à remarquer la grande similarité qui existe dans les caractères des maladies de textures semblables, quelle que soit la distance qui puisse séparer ces dernières; cela a procuré au pathologiste un avantage incalculable pour la recherche de la nature de la maladie: cela lui a permis de décrire les changements qui sont induits par cette nature de la maladie; de comparer ces résultats morbides avec les structures saines; et, enfin, de déterminer ces lois générales qui sont les seules bases rationnelles sur lesquelles le praticien peut fonder son diagnostic et son traitement. C'est à la connaissance de la structure que nous possédons maintenant, que devrait être attribué la production des nombreux excellents travaux qui ont été publiés à l'intérieur de quelques années et qui concernent la nature et les effets de la maladie, et l'on peut, dans une certaine mesure, reconduire à la même source la simplicité et les succès qui marquent aujourd'hui la pratique de la médecine ${ }^{16}$.

Une fois de plus, on a affaire ici à un auteur qui sait que les travaux de Smyth sont antérieurs à ceux de Pinel mais qui ignore que ce dernier, loin d'avoir inventé une problématique tissulaire parallèlement à et indépendamment de Smyth, a en fait repris les idées de ce dernier auteur. En outre, Grainger ignore l'apport capital de Haller et de Hunter pour la fondation de l'anatomie générale. Par contre, Grainger a vu que certaines conceptions histologiques attribuées ordinairement à Bichat, appartiennent en fait à A. Bonn. 
A la fin du XVIII ${ }^{e}$ siècle, A. Richerand (1779-1840) avait déjà affirmé ${ }^{17}$ avec force que Bichat s'était directement inspiré de Andreas Bonn (et de quelques autres) d'un bout à l'autre de son Traité des membranes (1799), dans la description des différents systèmes qu'elles forment.

Bonn donne, effectivement, dans l'ouvrage cité par Grainger, une description des divers systèmes de membranes que l'on peut considérer comme quasiment exhaustive par rapport à celle du Traité... de Bichat ${ }^{18}$. Il distingue quatre systèmes de membranes: 1) le système cutané, 2) le système muqueux, 3) le système fibreux, 4) le système séreux ${ }^{19}$.

Par exemple, Bonn donne une description très détaillée de chacune des membranes muqueuses: la tunique interne des paupières ou membrane conjonctive, la membrane qui revêt les cavités du nez ou membrane schneidérienne ${ }^{20}$, celle qui revêt l'intérieur de la bouche ${ }^{21}$, celle qui revêt l'intérieur de la gorge ${ }^{22}$; celle qui revêt le larynx ${ }^{23}$, celles du pharynx et de l'œsophage ${ }^{24}$, celle de l'estomac et des intestins ${ }^{25}$, celles de l'urètre, et de la vessie, du vagin et de l'utérus ${ }^{26}$.

Bonn donne aussi une analyse fouillée du système séreux ${ }^{26}$, du système fibreux ${ }^{27}$ et du système cutané ${ }^{28}$. Autre preuve, du fait que la problématique histologique est en voie de constitution avant les travaux des médecins de l'Ecole de Paris, c'est que l'ouvrage d'un auteur comme A. Bonn s'inscrit luimême dans le cadre d'une problématique que l'on trouve chez un auteur aussi important que Haller ${ }^{29}$.

Et, comme nous l'avons déjà montré, à la suite de certains auteurs de l'époque, c'est à «Haller qu'appartient incontestablement le mérite d'avoir analytiquement divisé le corps de l'homme en ses tissus constituants et d'avoir établi leurs propriétés physiologiques distinctives». ${ }^{30}$

Il est d'ailleurs particulièrement significatif que la place centrale de Haller dans la constitution de la problématique histologique ait été rétablie par Béclard à la section «Eléments anatomiques» de ses «Additions aux considérations générales» de l'Anatomie générale de X. Bichat:

Une opinion plus conforme à la nature des choses est celle qui reconnaît dans l'organisation trois éléments distincts: la fibre cellulaire, la fibre nerveuse, et la fibre musculaire. Des différences bien tranchées caractérisent ces trois tissus primitifs et ne permettent pas de les confondre. Tous les tissus organisés ont pour base l'une des ces trois fibres primitives ou résultent de l'association de plusieurs d'entre elles: ainsi l'élément cellulaire se retrouve dans la plupart des systèmes organiques;... Cette division des tissus primitifs indiquée par Haller et Blumenbach a été suivie par la plupart des anatomistes modernes ${ }^{31}$. 
Il nous semble donc que l'on peut dire qu'il y a eu, avant l'Ecole de Paris, deux types essentiels de contribution à la formation d'une problématique histologique. Le premier est celui des médecins et chirurgiens qui ont reconnu et fait fonctionner le principe de l'indépendance pathologique des tissus. Un auteur comme Bichat a dit lui-même que ce type de contribution à la problématique tissulaire avait précédé ses propres travaux:

Plusieurs médecins célèbres ont conçu cette vérité depuis Haller: ils ont senti que dans le système membraneux, diverses limites étaient à établir entre des organes jusqu'ici confondus. L'observation des caractères extrêmement variés que prend l'inflammation sur chaque membrane leur en a surtout indiqué la nécessité; ... ${ }^{32}$

A lire ce passage, on pourrait d'ailleurs se convaincre que Bichat fait ici référence directement à des auteurs comme Hunter, Smyth, Monro, Baillie, Vogel, Walter etc... et qu'il n'a pas vraiment eu besoin de lire la Nosographie philosophique de Pinel pour découvrir le principe fondamental d'une problématique histologique ${ }^{33}$.

Si un certain nombre des contributions de ce premier type à la problématique tissulaire peuvent apparaître ponctuelles ou limitées, par rapport à une problématique systématique des tissus comme celle de l'Ecole de Paris, on ne saurait en dire autant, selon nous, des contributions d'un deuxième type comme celles de Haller et de J. Hunter.

Nous avons déjà montré dans d'autres études que, avec Haller ${ }^{34}$ et surtout avec Hunter ${ }^{35}$, on est déjà en présence d'une problématique tissulaire générale et systématique. Plusieurs auteurs de l'époque avaient noté que la problématique d'une anatomie générale était déjà constituée chez Hunter et que ses conceptions étaient au fondement de tous les travaux de base de l'Ecole de Paris. C'est ainsi que A. Le Pelletier de la Sarthe a pu écrire:

\footnotetext{
«Dans son traité sur le sang, l'inflammation et les plaies d'armes à feu, communiqué d'après l'auteur, dès l'année 1762, publié en Angleterre en 1795, traduit en français en 1799, Hunter distingue déjà les phlegmasies d'après les tissus qu'elles occupent, émet des idées très lumineuses relativement à l'inflammation des parenchymes, et doit avoir servi de point de départ à Pinel, à Bichat, à Broussais lui-même pour la pensée mère de la nosographie philosophique, de l'anatomie générale et de la médecine physiologique» ${ }^{36}$.
}

Nous pensons donc pouvoir en conclure légitimement que la naissance, la constitution et la diffusion d'une problématique tissulaire se sont produites antérieurement et indépendamment de l'Ecole de Paris. 


\section{Notes}

1 Voir O.Keel, La généalogie de histopathologie. Une révision déchirante. Philippe Pinel, lecteur discret de James Carmichael Smyth. Préface de G. Canguilhem. Paris (1979), Vrin.

2 Voir O. Keel, «Les conditions de la décomposition (analytique) de l'organisme»: Haller, Hunter, Bichat, Les Etudes Philosophiques, $\mathrm{n}^{0} 1,1982$ : 37-62.

3 Ibid., voir aussi op. cit. note 1.

4 Voir O. Keel, «La pathologie tissulaire de J. Hunter», Gesnerus 37 (1980), fasc. 1/2: 46-61.

5 «John Hunter et Xavier Bichat. Les rapports de leurs travaux en pathologie tissulaire». Actes du XXVII Congrès International d'Histoire de la Médecine (1980), Barcelone. Impreso en Delfos, I.G. Esplugues de Llobregat. 1981. Tome II, pp.535-49. Voir aussi Keel, op. cit. (note 1); op. cit. (note 2), op. cit. (note 4).

6 Voir, par exemple, E.Ackerknecht, A Short History of Medicine. Revised edition. Baltimore et Londres, 1982, p.173sq.; J.Lesch, Science and Medicine in France. The Emergence of Experimental Physiology. 1790-1855, Cambridge et Londres 1984, p.67sq. F. Duchesneau, La physiologie des lumières. Empirisme, modèles et théories. The Hague, 1982, p. 434 sq. L'auteur, qui suit Bichat sur ce point sans remise en question, réitère la thèse qu'il n'y a pas encore d'analyse tissulaire chez Haller. (Voir sur ce point la note 34 ici.) Ce dernier aurait «négligé la spéficité de nature et de texture des fibres constituantes dans les diverses membranes et ignoré les propriétés vitales responsables de l'opération dynamique pour telle ou telle membrane», p. 434. Voir aussi J. Pickstone, "Bureaucracy, Liberalism and the Body in Post-Revolutionary France: Bichat's Physiology and the Paris School of Medicine". History of Science, XIX (1981): 115-142.

7 «Essai sur les ouvrages de Bichat, et spécialement sur les rapports qui existent entre les doctrines médicales de l'Anatomie générale, et celle de l'Examen des nouvelles doctrines», Journal complémentaire du Dictionnaire des sciences médicales (Paris 1822), vol.13, pp. 249-250. Souligné par nous.

8 Voir Keel, op. cit. (note 1).

9 De l'influence de l'anatomie pathologique sur les progrès de la médecine depuis Morgagni jusqu'à nos jours. Paris 1837, p. 98.

10 Voir Keel, «La constitution de la problématique de l'anatomie des systèmes selon Laennec». Laennec. 1781-1826. Collège de France. Paris 1981, Revue du Palais de la Découverte, Numéro spécial, pp. 189-207.

11 Ibid.

12 Op. cit., $3^{e}$ éd. Paris 1824, vol. 3, p. 230, souligné par nous. Voir aussi Keel, op. cit. (note 1).

13 Op.cit., Londres 1833, vol.2, p. 432.

14 Op.cit., p.4. Souligné par nous.

15 Op.cit. Eisenach 1822, p.23.

16 Op.cit. Londres 1829, pp.62-64. Souligné par nous. Ce passage nous a été signalé par le Pr. Pauline Mazumdar que nous voulons remercier ici.

17 Voir «Réflexions critiques sur un ouvrage ayant pour titre Traité des membranes par le C. Bichat» Magasin encyclopédique, vol. 6 an VIII (1799) pp. 260-72.

18 Voir A.Bonn, Specimen anatomico-medicum de continuationibus membranarum, Lugd. Bat. 1763. 
19 Ibid

20 Ibid. p.11 sq.

21 Ibid. p. 12.

22 Ibid. p. 15 sq.

23 Ibid. p. 16.

24 Ibid. p. 17 sq.

25 Ibid.

26 Ibid. p. 19 sq.

26 Ibid. pp. 29-52.

27 Ibid. pp. 21-29.

28 Ibid. pp.4-9. Sur tous ces points voir Keel, op. cit. (note 2), p.47 sq. Voir aussi O. Keel, «Aspects de la constitution du concept d'anatomie générale» Communication au XVI ${ }^{\mathrm{e}}$ Congrès International d'Histoire des Sciences (Bucarest 1981). Les travaux de A.Bonn sont analysés en détail dans cette étude.

29 Voir sur ce point Keel, op. cit. (note 2) p. 48 sq. et Keel, op. cit. (note 28).

30 Souligné par nous. Cité d'après J. Palmer, in Keel, op. cit. (note 1), p. 51 et in Keel, op. cit. (note 2), p.41. Palmer poursuit ainsi: «mais c'est à Hunter qu'on doit accorder celui d'avoir appliqué ce mode d'étude aux investigations pathologiques parce qu'il a compris que, comme chaque tissu est doué de propriétés particulières dans l'état de santé, de même il doit être affecté d'une manière spéciale par les causes des maladies, et conséquemment, que les mêmes modes de lésion doivent toujours produire des effets sembables sur tout les tissus analogues du corps».

31 Op.cit. Paris 1821, p.82, souligné par nous. Sur l'importance de Haller pour la problématique de l'anatomie générale, voir Keel, op. cit. (note 2), p. 43 sq.; P.-A. Béclard, Eléments d'anatomie générale, $3^{\mathrm{e}}$ éd., Paris 1852: «Haller a admis dans la composition des organes, outre le tissu cellulaire, formé par la réunion de fibres et de lames, et qui est le plus général et le plus répandu, la fibre musculaire et la substance médullaire. Cette division a été depuis assez généralement admise, avec quelques légères modifications plus ou moins heureuses. Ainsi Walther admet une texture membraneuse ou cellulaire; d'autres une cellulaire, une vasculaire, et une nerveuse; Pfaff une structure vasculaire, une fasciculaire et une cellulaire; d'autres une cellulaire, une vasculaire et une massive, ou sans cellule et sans vaisseaux. M. Chaussier a joint aux trois parties composantes de Haller une quatrième fibre, sous le nom de fibre albuginée; c'est la base des ligaments; M. Richerand y a joint la substance épidermique ou cornée. Parmi les vingt-et-un tissus admis par Bichat, il en est trois qu'il considère comme générateurs des autres, ce sont le cellulaire, le vasculaire et le nerveux. M. Meyer (Über Histologie, Bonn 1819) admet aussi trois organes élémentaires: 1 - la cellule, le vaisseau ou la glande, 2 - la fibre irritable, cellulaire ou musculaire, 3 - la fibre sensible ou le nerf», p. 96. Souligné par nous. Comme on le voit, à certaines modifications près, le système des trois tissus générateurs de Bichat peut être ramené à celui de Haller. Plus tard, Meyer rectifie Bichat et reprend intégralement le système de Haller dans le premier traité d'histologie qui soit paru. Pour l'étude et l'analyse des tissus chez Haller voir surtout Elementa physiologiae corporis humani. 8 vols. Berne/Lausanne 1757-1766.

32 Traité des membranes. Edition F. Magendie. Paris 1827, p. 4.

33 Voir sur ce point Keel, op. cit. (note 4), p. 47 sq. 
34 Voir Keel, op. cit. (note 11), et Keel op.cit. (note 2). Quelques auteurs ont indiqué en passant, mais sans le dégager assez explicitement, que Haller a rendu possible la problématique histologique. Voir K.E. Rothschuh, Geschichte der Physiologie, Berlin 1953, p.77; F.Berg, Die Lehre von der Faser als Baustein des Organismus, Klin. Wochenschrift, $\mathrm{n}^{\circ} 44,1939$, p.1427, et p.1429; R.Schär, Albrecht von Hallers neue anatomisch-physiologische Befunde und ihre heutige Gültigkeit, Berne 1958, p.5; E. Hintzsche: «Haller», Dictionary of Scientific Biography, vol. VI, p. 62.

35 Voir Keel op. cit. (note 1); Id, op. cit. (note 2) et «J. Hunter et X. Bichat. Les rapports de leurs travaux en pathologie tissulaire», op. cit. (note 5). Dans ce texte, nous avons donné les références à plusieurs séries de passages de Hunter sur les différents systèmes tissulaires et nous avons montré que non seulement il y a isomorphisme, à ce niveau, entre la problématique de Hunter et celle de Bichat, mais encore que Bichat s'est approprié cette problématique de Hunter. Voir, en particulier pp.536-540.

36 Histoire de la révolution médicale du XIX siècle, Paris 1854, p. 19.

\section{Abstract}

How was the problematic of histology constituted? According to the thesis that has long been accepted in the history of medicine, the formation of this problematic was the work of the Paris School of Clinical Medicine (Pinel, Bichat and their students). We will show that an important number of late eighteenth and early nineteenth century authors were not of this opinion and that, at the time, it was known that the histological problematic had appeared in the works of other authors (such as A.von Haller, A. Bonn, J.C.Smyth, J. Hunter, etc...) and had been developed by the latter before being adopted by the members of the Paris Clinical School.

\section{Prof. Othmar Keel}

Département d'Histoire

Université de Montréal

Case postale 6128, Succursale A

Montréal (Québec)

Canada 